\title{
Delayed Development of Traumatic Life-Threatening Acute Subdural Hematoma and Its Management: Challenge for Neurosurgeon - A Case Report and Review of Literature
}

\author{
Pratap Chandra Nath ${ }^{1}$ Sudhansu Sekhar Mishra ${ }^{1}$ Somnath Prasad Jena ${ }^{1}$ Soubhagya Ranjan Tripathy ${ }^{1}$ \\ ${ }^{1}$ Department of Neurosurgery, Shri Ramachandra Bhanj Medical \\ College, Cuttack, Odisha, India \\ Address for correspondence Pratap Chandra Nath, MCh, Department \\ of Neurosurgery, Shri Ramachandra Bhanj Medical College, Cuttack, \\ Odisha 753007, India (e-mail: drpratapnath@rediffmail.com).
}

Indian J Neurosurg 2016;5:207-212.

\begin{abstract}
Delayed development of traumatic acute subdural hematoma in patients having a coagulation disorder with or without taking anticoagulants have been well reported in literature. But delayed development of acute subdural hematoma (ASDH) in patients with head injury without any coagulation disorder is rare as per our knowledge. In this case report, we are reporting a 28-year-old male patient, who developed delayed posttraumatic life-threatening ASDH after 48 hours of trauma. The patient had no coagulation disorder. He was not an alcoholic and all his examinations, including coagulation profile, liver function tests, and peripheral smear examination were within the normal limits. We want to highlight that mere absence of a lesion on computed

Keywords

- delayed

- life-threatening ASDH

- rete vasorum tomography and negative clinical, with radiological finding, at an emergency department, does not always hold the eligibility to discharge a head injury patient. Observation for at least 24 hours with subsequent minimum one short interval followup is necessary for the safety of the patient.
\end{abstract}

\section{Introduction}

Minor traumatic head injury as per the Glasgow coma scale (GCS) system is not always literally minor. The mere absence of a lesion on computed tomography (CT) and negative clinical findings does not always hold the eligibility to discharge a head injury patient from an emergency department. Though most of the minor head injury patients show uneventful good recovery, but a very small number of patients develop life-threatening intracranial complication needing neurosurgical intervention.

Though in many literatures, delayed intracranial hematoma have been described in patients with coagulation disorder or patients on anticoagulation therapy, this is a rare occurrence in a patient, such as our case, without having coagulation disorder or any other risk factors. Most of the literature has been described regarding delayed development of traumatic

received

March 8, 2015

accepted after revision

December 18, 2015

published online

June 8, 2016
DOI http://dx.doi.org/ 10.1055/s-0036-1581986. ISSN 2277-954X. intraparenchymal or extradural hemorrhages. ${ }^{1-4}$ But delayed development of acute subdural hematoma (ASDH) has been a rarely reported entity. Here, we are reporting a 28 -year-old male patient who had sustained minor head injury without any clinical or radiological abnormality initially and discharged from an emergency department after 18 hours of trauma. After 48 hours, he developed ASDH for which he was readmitted. The patient's condition deteriorated day by day, for which he was operated on the 15th posttrauma day. Here, we discuss its pathogenesis, management, outcome, and review of the related literature.

\section{Case Report}

A 28-year-old male patient sustained head injury due to a fall from the bike. He presented to an emergency department after 8 hours of trauma being referred from a (c) 2016 Neurological Surgeons' Society of India

License terms

(c) $(1) \$$ 
primary hospital. He had one episode of vomiting, during treatment at the primary care hospital. He had undergone CT scan of brain at approximately 9 hours and 30 minutes of trauma at our emergency department (-Fig. 1). On initial examination, he had no loss of consciousness, had one episode of vomiting, no nasal or ear bleeding, no convulsion; his vitals were stable, pupil were bilateral normal, and GCS was E4V5M6. On CT scan of the brain, no bony fracture, intracranial lesion, edema, or mass effect were found. The patient, after observation for another 6 hours, was discharged from the emergency department.

After 48 hours of trauma, he became disoriented with the complaint of headache and was readmitted to the emergency department with a GCS of E4V4M6. Immediately after primary care, the patient had undergone a repeat CT scan of the brain which showed right-sided ASDH with midline shift and mass effect ( - Fig. 2). On further investigation, his coagulation profile and peripheral smear comment were normal. He was not a known alcoholic, had no history of recent blood transfusion, and was not a known case of hypertension, diabetes, tuberculosis, sickle cell anemia, or thalassemia. He was first planned for conservative management. He underwent four CT scans, every 72 hours, to find the progression (-Figs. 3 and $\mathbf{4}$ ). During the entire period, the patient presented with severe, intense headache, pulse within 60 to 70/minutes, and disorientation with irrational talking, and other different psychological features.

Due to intense headache, evacuation of $120 \mathrm{~mL}$ of semisolid blood clots done by an osteoplastic flap ( - Figs. 5 and $\mathbf{6}$ ). Dural enhancement was done with G-dura patch (G. Surgiwear Ltd, Rasoolpur Jahanganj, Sahajahanpur, Uttar Pradesh, India) and bone apposed in a hinged manner to combat any further brain swelling. Postoperative CT scan on the 5th day showed no mass effect and hinged apposed bone in three dimensional reconstructed views (-Figs. 7 and 8). The patient was discharged on the 7th postoperative day with GCS scores of 15/15 and Glasgow outcome scale of 5 (GOS).

\section{Discussion}

Delayed traumatic intracranial hemorrhage (DTICH) was described in many literatures previously. ${ }^{1-9}$ Most were intraparenchymal and extradural hematoma, but the delayed development of ASDH is a rarely reported entity. ${ }^{1-8}$ Hardly five to six cases have been reported yet. First delayed intracranial hemorrhage was reported in 1891 by Bollinger as Spät-apoplexie, who coined the term "traumatische Spät-Apoplexie" and described four patients of DTICH, of which one patient died after the apoplectic event. ${ }^{1}$ As per Diaz et al, DTICH is diagnosed if there is: (1) Initial injury occurred with the head in motion. (2) Patients with transient or permanent loss of consciousness. (3) Focal neurological sign or skull fracture. (4) Hematoma occurring less than 2 weeks postinjury. ${ }^{2}$ But Gudeman et al defines the DTICH as subsequently developed high-density parenchymal lesion in an area previously normal or with negligible abnormality. ${ }^{3}$ Fukamachi et al describes hematoma that appears

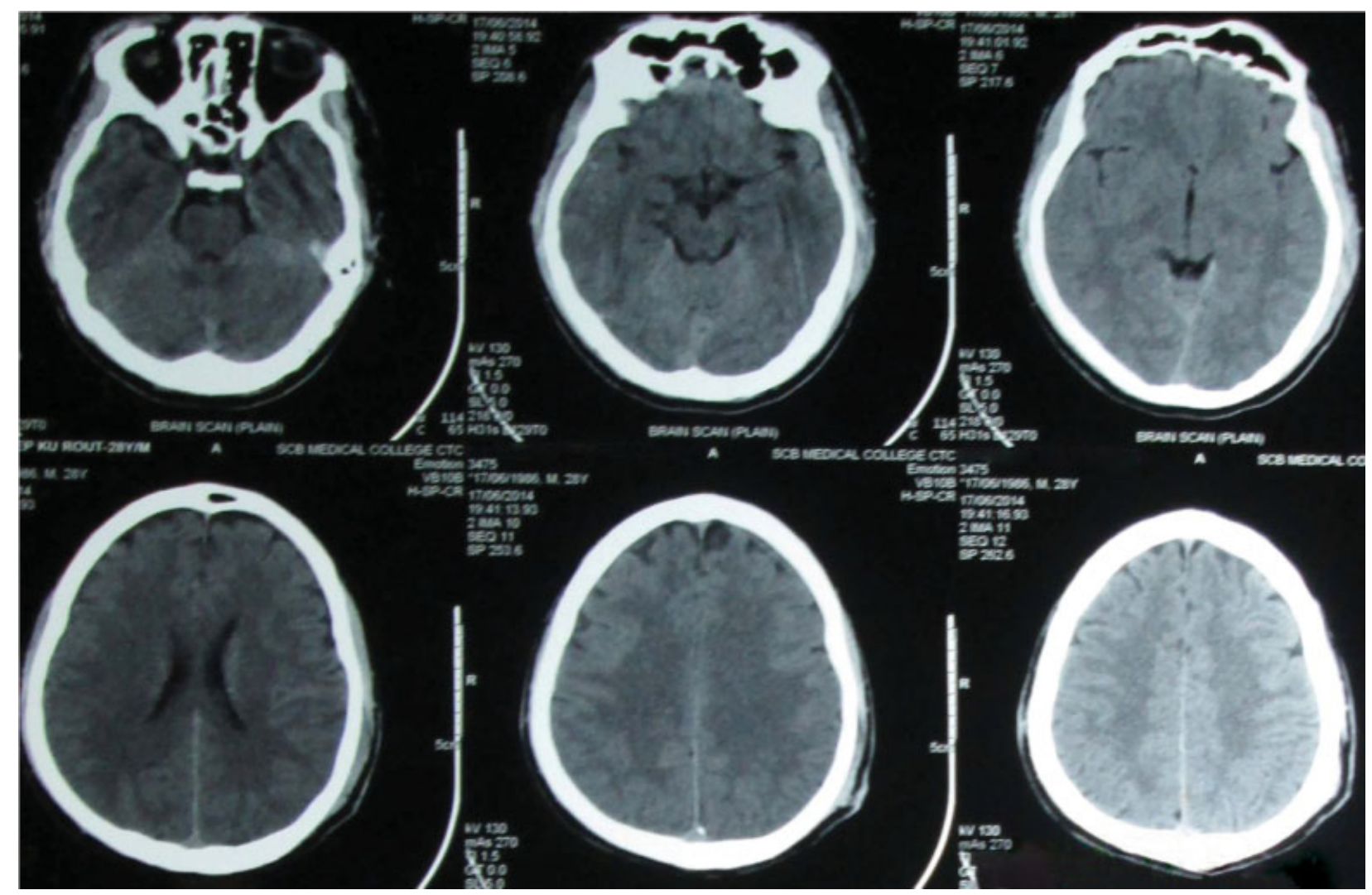

Fig. 1 The initial CT scan showing no intracranial pathology or mass effect. CT, computed tomography. 


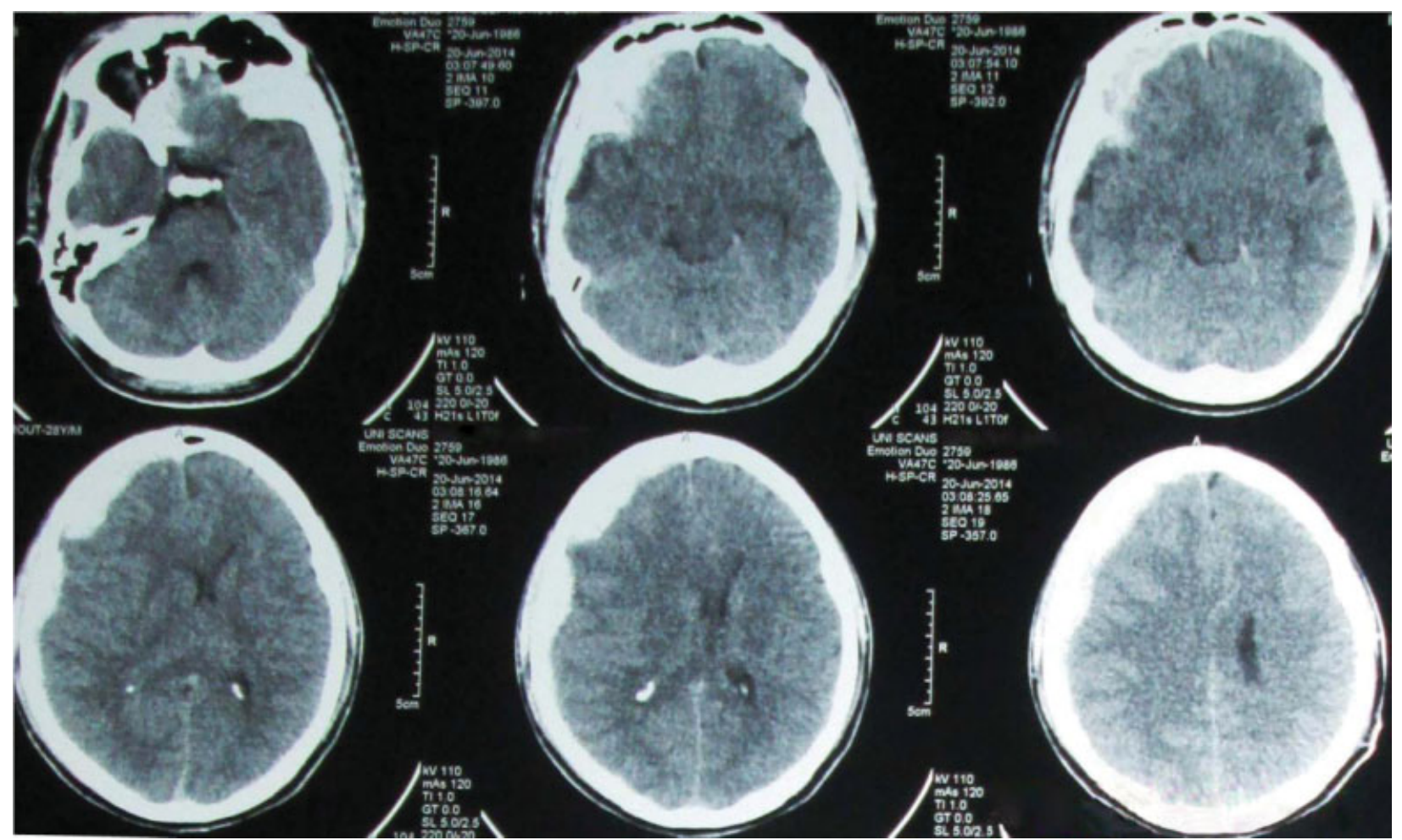

Fig. 2 The CT scan done after 48 hours showing right-hemispheric ASDH with mass effect. ASDH, acute subdural hematoma; CT, computed tomography.

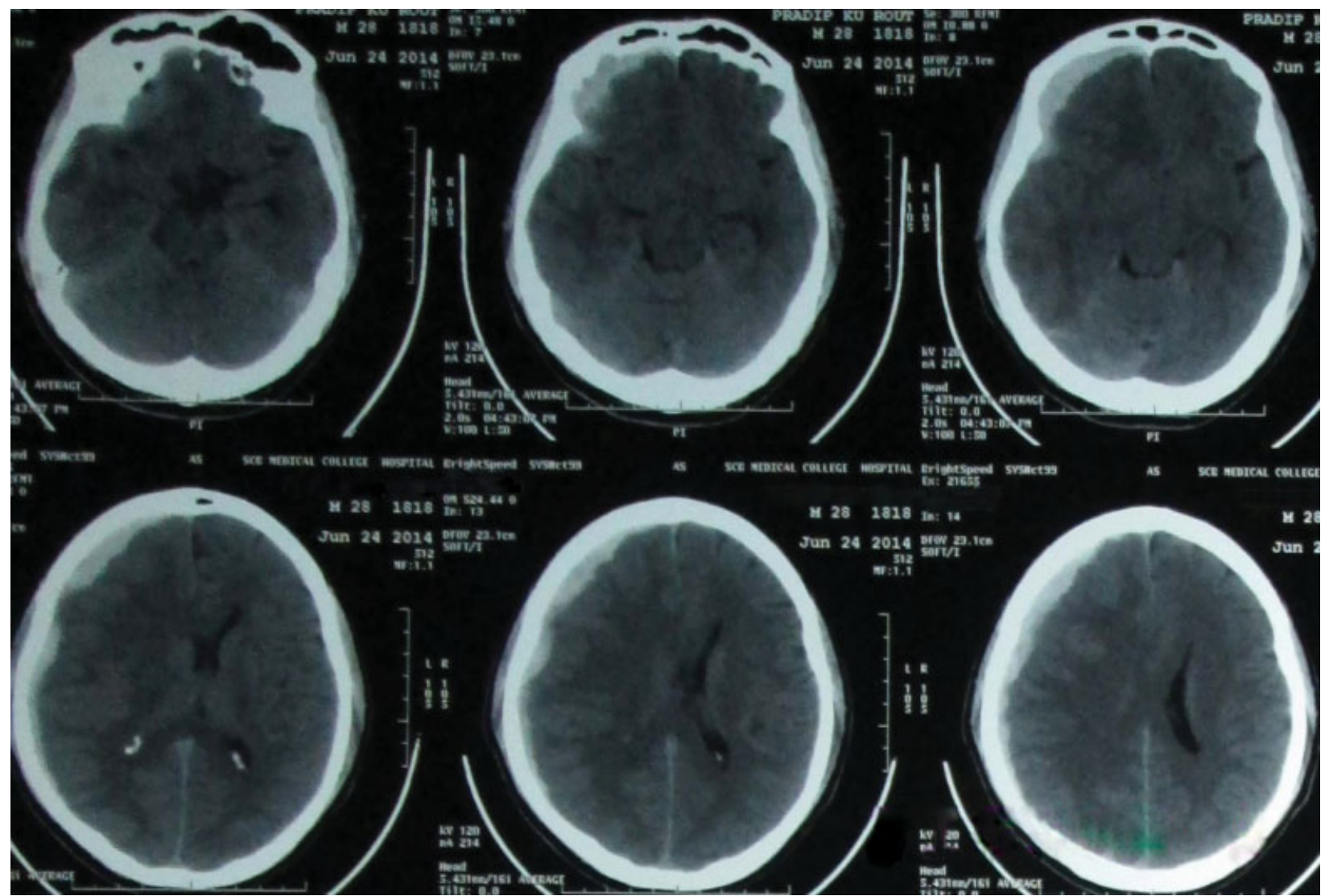

Fig. 3 The $\mathrm{CT}$ scan done on the 7th day of trauma showing right-hemispheric ASDH with mass effect. ASDH, acute subdural hematoma; $\mathrm{CT}$, computed tomography. 


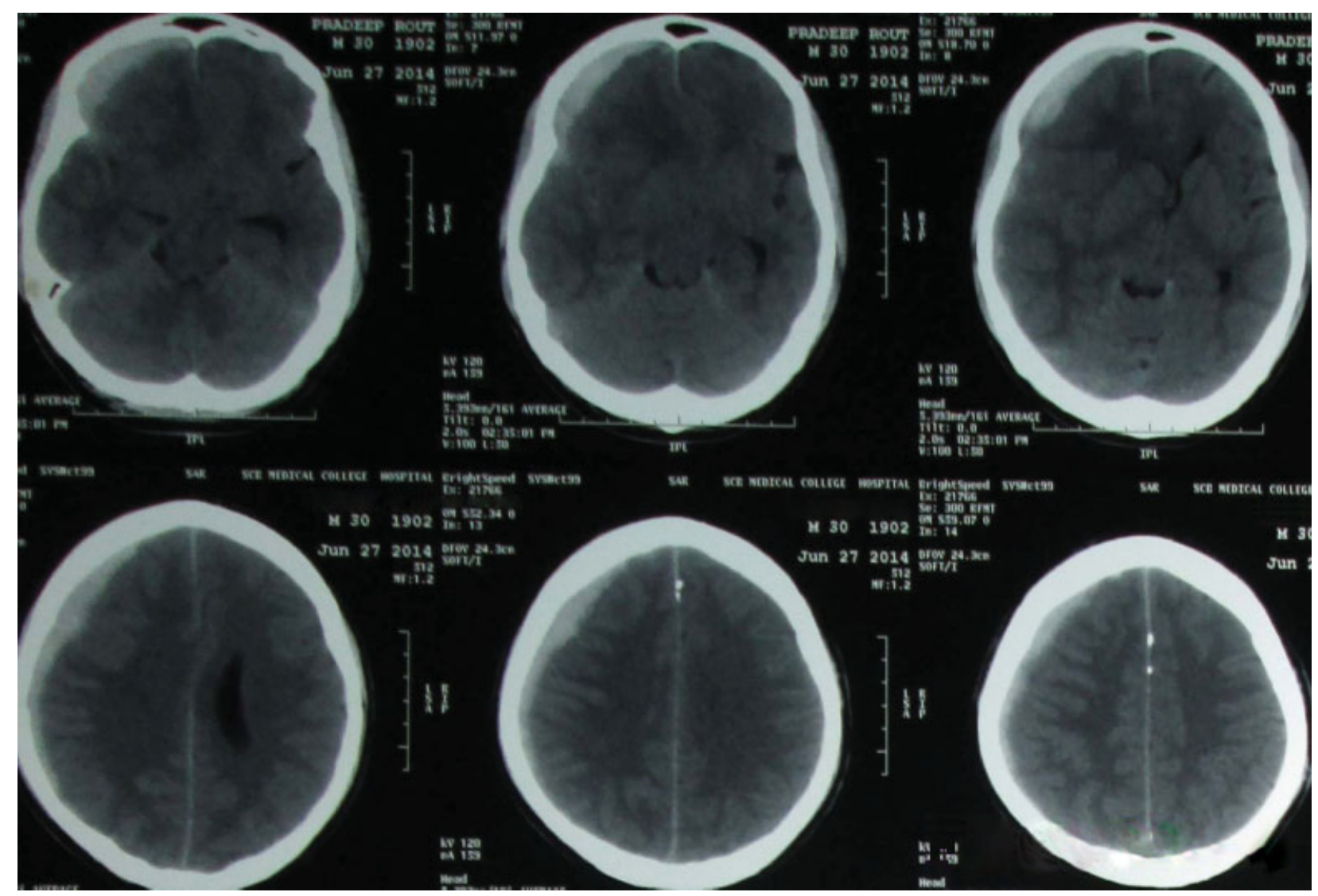

Fig. 4 The CT scan done on the 9th day of trauma showing right-hemispheric ASDH with mass effect. ASDH, acute subdural hematoma; CT, computed tomography.

subsequently, where the initial CT scan could not elicit any abnormalities. ${ }^{9}$

The pathogenesis of delayed intracranial hematoma is still not well understood. Patient with risk factors, such as coagulation disorder, taking anticoagulants, alcoholism, deranged liver function, chronic illnesses, such as diabetes mellitus, hypertension, underlying cardiac disease, etc., or any underlying intracranial lesion, such as a tumor or aneurysm, are prone for intracranial hemorrhage and may

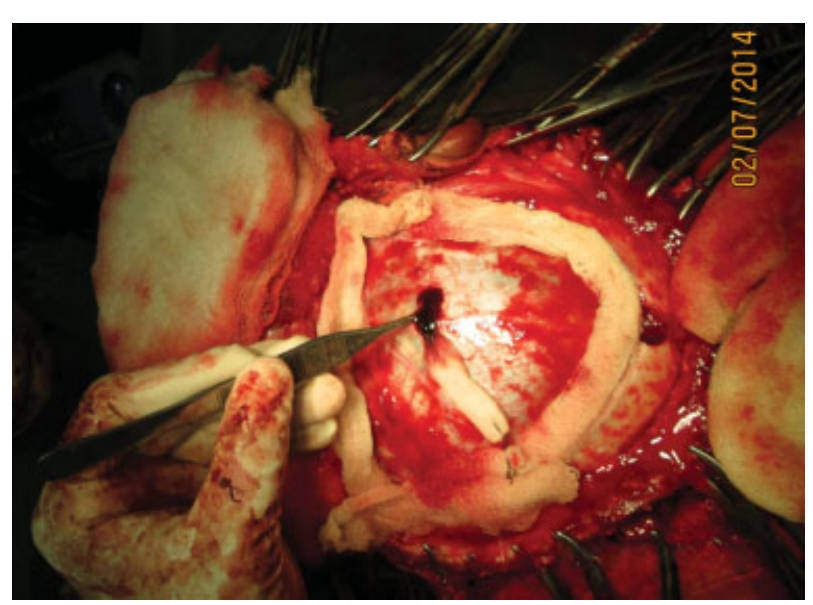

Fig. 5 Intraoperative photo showing under pressure subacute SDH leaking on the nick of the dura. SDH, subdural hematoma. occur after a few days of trauma or detected coincidentally. Our patient was not an alcoholic and was not associated with any of the above risk factors for bleeding. His initial CT scan done after 9 hours of trauma was absolutely normal and the patient was discharged from casualty after 18 hours of trauma. But after 48 hours, CT scan showed hemispheric ASDH. There was no history of further trauma within this significant 18 to 48 hours. This creates pathological dilemma, as the probable etiopathogenesis described by

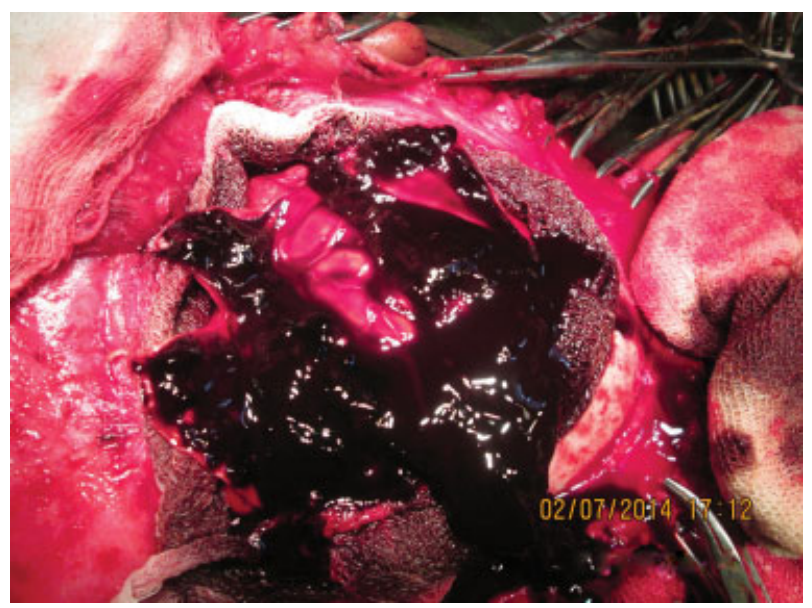

Fig. 6 Intraoperative photo showing semisolid blood clots (around $120 \mathrm{~mL}$ ). 


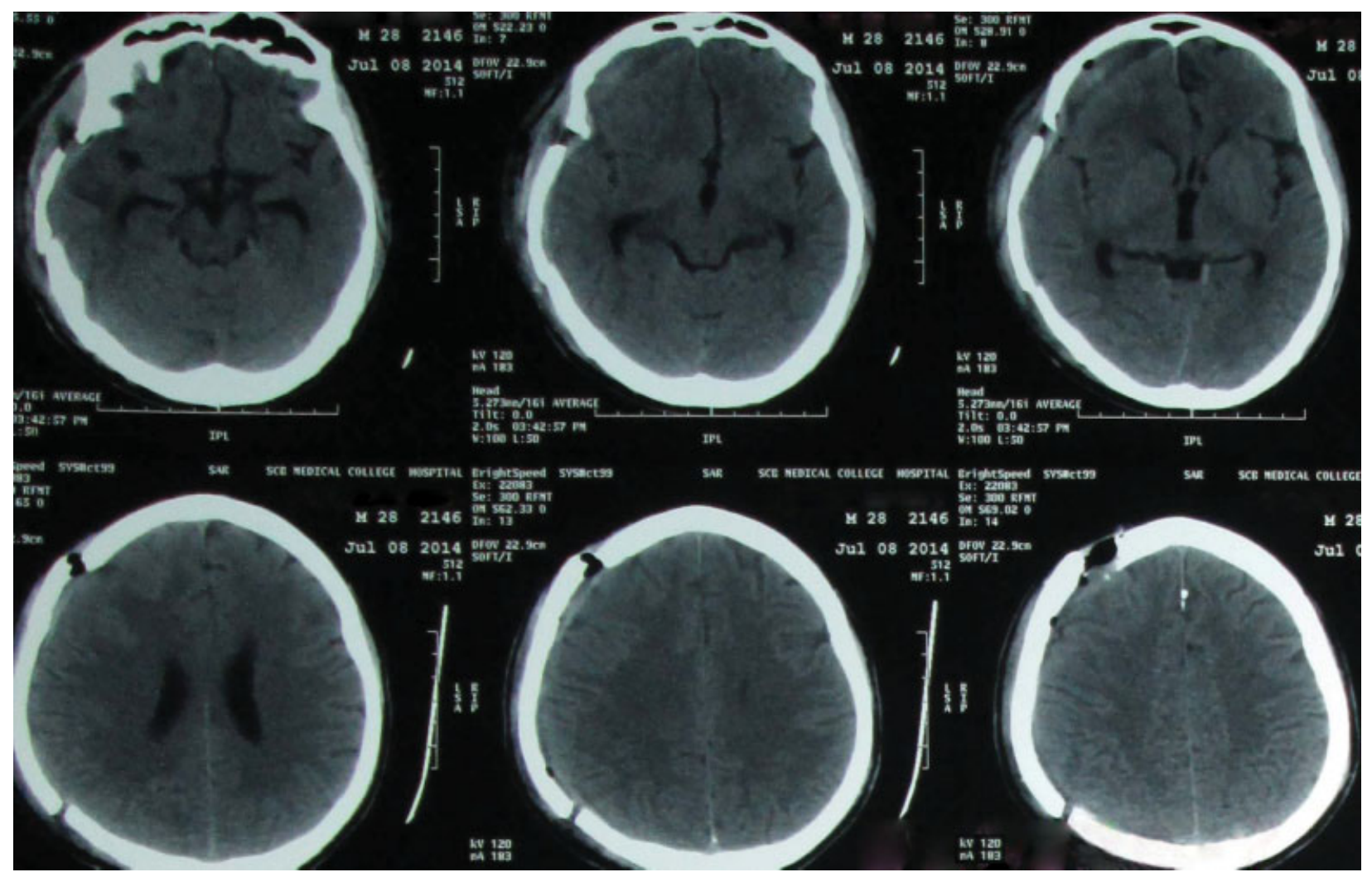

Fig. 7 Postoperative CT scan on day 5, showing no residual subdural hematoma and no mass effect. CT, computed tomography.

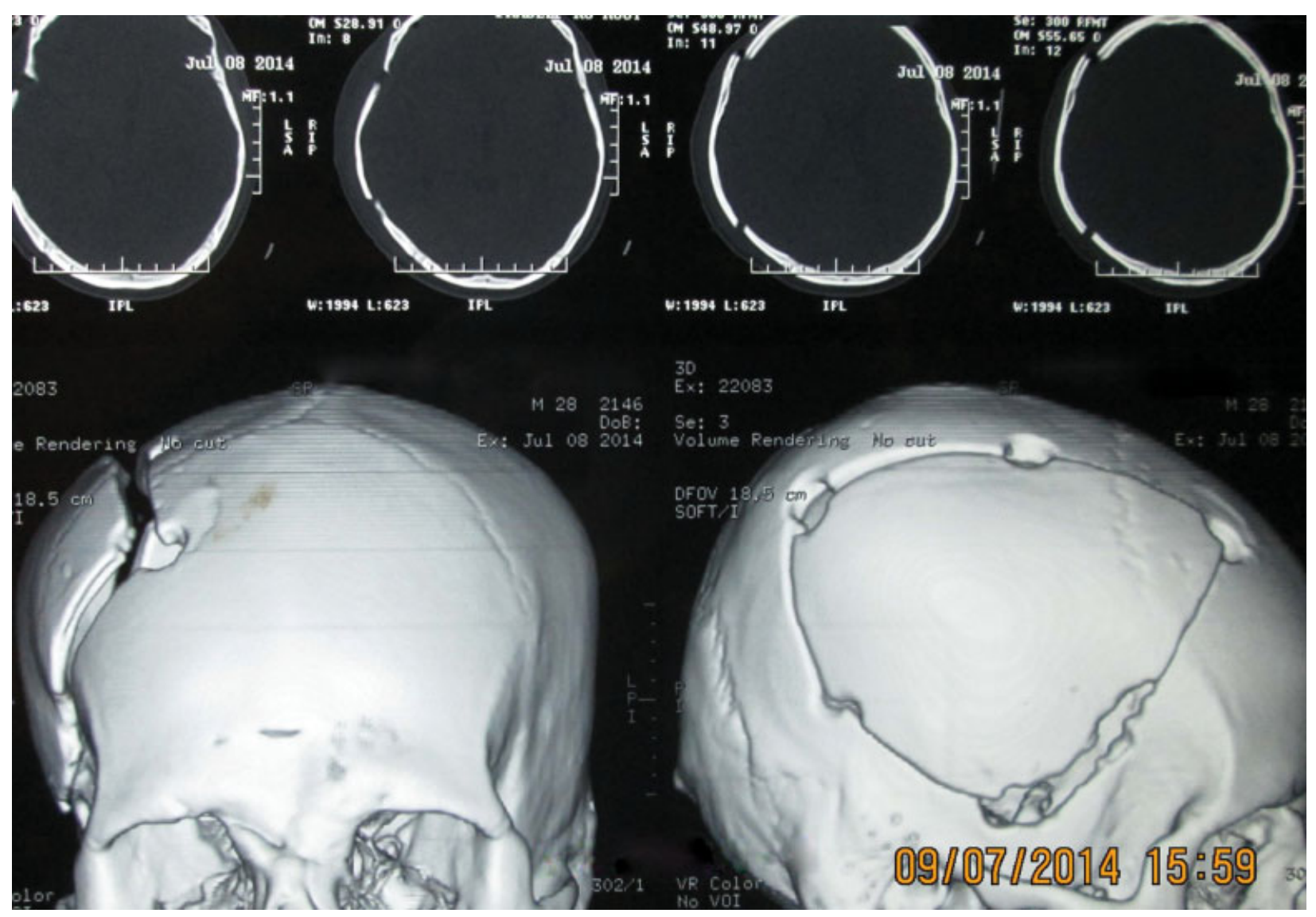

Fig. 8 Postoperative 3D reconstructed skull showing a hinged osteoplastic flap and calvarial defect. 3D, three-dimensional. 
many articles regarding delayed development of hematoma seems to be inapplicable here. Many of them described the fact with the patient having risk factors. The only hypothesis that can be suggested in our case is shearing of the brain within the cranial cavity resulting in microvascular injury of the bridging vein wall; which later due to surrounding degenerative changes caused by hypoxia of the vessel wall, resulted in necrosis followed by disruption of vessel wall and subsequent bleeding. As described by Zervas et $\mathrm{al}^{10}{ }^{10}$ the cerebral blood vessels are devoid of vasa vasorum. The cerebral vessels contain a rete vasorum in the adventitia that is permeable to large proteins and is in continuity with the subarachnoid space. This substructure may be analogous to the systemic vasa vasorum and may contribute to the nutrition of the cerebral blood vessels. It can be suggested here that injury to this rete vasorum may cause hypoxic injury to the vessel wall, leading to degeneration and disruption of the vessel wall, with subsequent bleeding which needs further histopathological evaluation. The other hypotheses proposed were spontaneous vascular rupture, resulting from progressive vascular degeneration, ${ }^{1}$ the local metabolites causing vascular wall injury, ${ }^{2}$ vasoparalysis resulting from local hypoxia, ${ }^{3}$ and increased intracranial pressure causing venous congestion produced by Valsalva effect. $^{7}$

In this type of ASDH, the ideal surgical management should be decompressive craniectomy with evacuation of ASDH. But we had done hinge osteoplastic decompressive craniotomy instead of decompressive craniectomy in spite the brain was falling without visible pulsation. The recovery was good and patient discharged with GCS of $15 / 15$ and GOS of 5 . As presented in this case, the clinical observation for 24 hours should be accompanied by at least three follow-ups at the $3 \mathrm{rd}$, 7th, and 14 th posttrauma day; even if the head injury is minor or having an initial normal CT scan to overcome these neuropathological dilemmas.

\section{Conclusion}

Minor traumatic head injury as per the GCS system is not always literally minor. The mere absence of any CT finding should not hold the eligibility for discharge of a patient from an emergency department. Hospital observation of all head injury patients for at least 24 hours should be done. All patients on discharge from an emergency department should be warned regarding features of further deterioration. It may further be advocated that at least three follow-ups at the 3rd, 7 th and 14 th day, should be done even if the head injury is minor or having an initial normal CT scan, for the benefit of patients. Subsequent CT scans need an opinion of the treating neurosurgeon to avoid excessive exposure to radiation. Timely intervention yields excellent results in delayed traumatic ASDH (DTASDH).

Conflict of Interest

All authors have none to declare.

\section{References}

1 Bollinger O. Uber traumatische Spat-Apoplexie: Ein Beitrag zur lehre von der Hirnerschutterung. In Internationale Beitrage Zur Wissenschlaftlichen Medizen. Festschrift, Rudolf Virchow (eds.). A. Hirschwald, Berlin;1891:457-470

2 Diaz FG, Yock DH Jr, Larson D, Rockswold GL. Early diagnosis of delayed posttraumatic intracerebral hematomas. J Neurosurg 1979;50(2):217-223

3 Gudeman SK, Kishore PRS, Miller JD, Girevendulis AK, Lipper $\mathrm{MH}$, Becker DP. The genesis and significance of delayed traumatic intracerebral hematoma. Neurosurgery 1979;5(3): 309-313

4 Nelson AT, Kishore PR, Lee SH. Development of delayed epidural hematoma. AJNR Am J Neuroradiol 1982;3(5): 583-585

5 Cassin BJ, Spitz WU. Concentration of alcohol in delayed subdural hematoma. J Forensic Sci 1983;28(4):1013-1015

6 Koumtchev Y, Petkov S, Gozmanov G. A case of delayed subdural hematoma. Folia Med (Plovdiv) 1994;36(2):59-61

7 Matsuda W, Sugimoto K, Sato N, Watanabe T, Fujimoto A, Matsumura A. Delayed onset of posttraumatic acute subdural hematoma after mild head injury with normal computed tomography: a case report and brief review. J Trauma 2008; 65(2):461-463

8 Chen S, Xu C, Yuan L, Tian H, Cao H, Guo Y. Fatal deterioration of delayed acute subdural hematoma after mild traumatic brain injury: two cases with brief review. Chin J Traumatol 2014;17(2): 115-117

9 Fukamachi A, Nagaseki Y, Kohno K, Wakao T. The incidence and developmental process of delayed traumatic intracerebral hematomas. Acta Neurochir (Wien). 1985;74:35-39

10 Zervas NT, Liszczak TM, Mayberg MR, Black PM. Cerebrospinal fluid may nourish cerebral vessels through pathways in the adventitia that may be analogous to systemic vasa vasorum. J Neurosurg 1982;56(4):475-481 